\title{
EFETIVIDADE DA RIZOTOMIA FACETÁRIA POR RADIOFREQUÊNCIA NA LOMBALGIA MECÂNICA CRÔNICA
}

\author{
EFFECTIVENESS OF RADIOFREQUENCY FACET RHIZOTOMY IN THE TREATMENT OF \\ MECHANICAL BACK PAIN
}

EFECTIVIDAD DE LA RIZOTOMÍA FACETARIA EN LUMBAGO MECÁNICO

Lourimar Octaviano de Tolêdo ${ }^{1}$, Sebastião Maurício Macedo ${ }^{2}$, Tarciso Fávaro ${ }^{3}$

\begin{abstract}
RESUMO
Objetivo: Avaliar a efetividade da rizotomia facetária lombar por radiofreqüência no tratamento da dor lombar mecânica. Métodos: Estudo prospectivo de 23 pacientes tratados com rizotomia por radiofrequência devido à dor lombar mecânica. O registro da intensidade da dor foi medido através de pontuação pela escala visual analógica (EVA) e o número de analgésicos administrado a cada paciente no pré-operatório e nos controles de 3, 6 e 12 meses de pós-operatórios. Resultados: Foi observado um declínio significativo da intensidade da dor $(p<0,0001)$ nos pacientes estudados. Conclusões: Na série de pacientes avaliados, o tratamento foi efetivo no alívio sintomático da dor lombar mecânica e determinou menor uso de analgésicos após a realização do procedimento.
\end{abstract}

Descritores: Rizotomia; Dor lombar; Articulação zigoapofisária; Resultado de tratamento.

\begin{abstract}
Objective: To evaluate the effectiveness of radiofrequency lumbar facet rhizotomy in the treatment of mechanical low back pain. Methods: Prospective study of 23 patients treated with radiofrequency rhizotomy in mechanical low back pain. The pain intensity was measured by the visual analog scale (VAS) and the number of analgesics administered for each patient preoperatively and 3, 6 and 12 months postoperatively. Results: We observed a significant decline in pain intensity $(p<0.0001)$ in patients. Conclusions: In the series of patients assessed the treatment was effective in relieving symptoms of mechanical low back pain and the use of analgesics after the procedure was lower.
\end{abstract}

Keywords: Rhizotomy; Low back pain; Zygapophyseal joint; Treatment outcome

\section{RESUMEN}

Objetivo: Evaluar la efectividad de la rizotomía por radiofrecuencia facetaria lumbar en el tratamiento del lumbago mecánico. Métodos: Estudio prospectivo de 23 pacientes tratados con rizotomía por radiofrecuencia en lumbago mecánico. El registro de la intensidad del dolor se midió por la puntuación de la escala analógica visual (VAS) y el número de analgésicos administrados a cada paciente antes de la operación, y 3, 6 y 12 meses después de la intervención. Resultados: Se observó una disminución significativa en la intensidad del dolor ( $p<0,0001)$ en los pacientes. Conclusiones: En la serie de pacientes evaluados el tratamiento fue efectivo en el alivio de los síntomas del lumbago mecánico e indicó un menor uso de analgésicos después del procedimiento.

Descriptores: Rizotomía; Dolor en la región lumbar; Articulación cigapofisaria; Resultado del tratamiento.

\section{INTRODUÇÃO}

As articulações facetárias apofisárias da coluna vertebral são articulações diartrodiais típicas, que estão sujeitas aos mesmos processos degenerativos observados nas outras articulações do corpo. O conceito de que as articulações facetárias poderiam ser fonte de dor foi introduzido na década de 1940 por Badgley 1 , o qual demonstrou a inervação neural para o complexo articular facetário, através da dissecção cadavérica. A atual compreensão da artropatia articular facetária e sua contribuição para a gênese da dor lombar na fase de envelhecimento continuam evoluindo.

A síndrome facetária continua sendo uma entidade clínica precariamente definida. Admite-se que os sintomas incluem dor localizada na região lombar com possível irradiação para as extremidades, rigidez na articulação lombo-sacra, hipersensibilidade para-lombar e ausência de déficits neurológicos ${ }^{2-4}$. No início desse processo, as radiografias podem revelar alterações mínimas ou ter um aspecto completamente normal ${ }^{5}$. O diagnóstico deverá ser feito clinicamente e será confirmado pela injeção de anestésico local no interior da articulação sob orientação fluoroscópica ${ }^{6,7}$.
A dor crônica da coluna vertebral incapacita enormemente a qualidade de vida de seus portadores. Quando há refratariedade aos tratamentos clínicos e de reabilitação, métodos cirúrgicos se fazem necessário.

A rizotomia facetaria lombar por radiofrequência percutânea foi proposta por Shealy em $1975^{8}$. Desde então, esse método vem largamente sendo empregado, com sucesso na literatura mundial. A técnica consiste na introdução de uma agulha na região dorsal do paciente sob controle radiográfico, posicionando a ponta da agulha nas articulações facetárias da coluna lombar. As estruturas sensitivas facetárias da coluna lombar são lesadas por radiofrequência e assim, é obtido um efetivo controle da dor lombar de forma minimamente invasiva ${ }^{8-10}$.

Existe evidência sobre a efetividade desta técnica no alivio da dor lombar mecânica crônica,especialmente no alívio a curto pra$\mathrm{zo}^{11-13}$. Entretanto, a eficácia depende de um diagnóstico correto e posicionamento adequado do eletrodo ${ }^{14}$

O objetivo do presente estudo é avaliar a efetividade no alívio da dor lombar mecânica com a realização da rizotomia lombar percutânea por meio de radiofrequência.

1. Ortopedista do Grupo de Coluna Vertebral do Hospital Metropolitano - Serra, ES,Hospital Santa Rita -Vitória,ES Brasil.

2. Chefe do Serviço de Ortopedia eTraumatologia do Hospital Metropolitano - Serra, ES, Brasil.

3. Ortopedista do Grupo de Coluna Vertebral do Hospital Metropolitano -Serra, ES, Hospital Santa Rita - Vitória, ES, Brasil. 


\section{MÉTODOS}

Características do estudo - Série prospectiva e consecutiva de 23 pacientes submetidos à rizotomia facetária lombar por radiofreqüência entre 2007 e 2008. A população era composta por seis pacientes do sexo masculino (26,09\%), com idade entre 27 e 62 anos, e média de 41,1 anos; e 17 pacientes do sexo feminino $(73,91 \%)$ com idade entre 32 e 64 anos e média de 51,6 anos .A variação total de idade foi de 27 a 64 anos com média de 47 anos. Destes um melanodérmico (4,34\%). O período de sintomas pré-operatórios variou de 2 a 12 anos. O diagnóstico foi clínico e em todos os casos usamos radiografia dinâmica e ressonância magnética da coluna lombo-sacra. O diagnóstico foi confirmado com a injeção de um anestésico local o interior da articulação sob orientação fluoroscópica. Todos os pacientes foram tratados com administração de AINE, analgésicos convencionais ,opioides e fisioterapia antes da indicação do procedimento cirúrgico. A avaliação pré e nos 3,6 e 12 meses de pós-operatório foi realizada por meio da escala visual analógica de dor (EVA) e uso de medicamentos.

\section{Descrição da técnica cirúrgica}

Com o paciente posicionado em decúbito ventral sob sedação anestésica leve, sem curarizar (citrato de fentanila e diazepam) e anestésico local (lidocaína $2 \%$ sem vasoconstrictor) no trajeto das agulhas a serem introduzidas. Sob controle fluoroscópico, a cânula é colocada na faceta apropriada. O eletrodo é inserido através da cânula e o cabo de conexão do eletrodo é conectado no gerador de radiofrequência. É realizada a medição da impedância, que deve estar entre 300 e 450 Homes. O gerador de radiofrequência é programado no modo estimulação a $20 \mathrm{~Hz}$ e $1 \mathrm{~ms}$ e eleva-se lentamente a tensão de 2 a 3 V.O paciente não deve apresentar movimentos involuntários. As facetas são lesadas utilizando a temperatura entre $80-90^{\circ} \mathrm{C}$ durante 60 segundos. Após a realização da lesão por radiofrequência utiliza-se $10 \mathrm{ml}$ de solução com anestésico (bupivacaína 0,50\% sem vasoconstrictor) por nível. Não foram administrados corticóides. Os procedimentos duraram entre 30 e 75 minutos (média de 52,5 minutos), com internação inferior a $24 \mathrm{~h}$ e retorno ao trabalho em até 15 dias. O gerador de radiofrequência utilizado no presente estudo foi o RF GENERATOR V3 (MULTI-RF) e os kits descartáveis de eletrodos para ablação facetária foram OPTIMA TM PROBE, ambos da BAYLIS MEDICAL (MONTREAL,QC,CANADA)

\section{Seguimento clínico}

Os pacientes foram controlados no pré-operatório e nos 3,6 e 12 meses de pós-operatórios, registrando-se o número de analgésicos empregados e a intensidade da dor medida através da escala visual analógica (EVA).

\section{Análise estatística}

As variáveis contínuas foram descritas e expressadas em valores absolutos. Para comparar o número de analgésicos empregados e a pontuação da escala visual analógica entre o pré-operatório e nos 3,6 e 12 meses de pós-operatório, sendo empregada a escala de Friedman. Foi considerada como estatisticamente significativo valores de p menores que 0,05 . Todos os cálculos foram realizados no software SPSS (SPSS inc.,Chicago,IL).

\section{REFERÊNCIAS}

1. Badgey CE. I. The importance of the lumbo-sacral joint in low back pain with sciatic radiation. II. A new theory to explainradiation of pain. In: Presented at the annual meting of the American Academy of Orthopaedic Surgeons, St. Louis; 1936.

2. Frymoyer JW, Moskowitz RW. Spinal degeneration: pathogenesis and medical management. In: Frymoyer JW. The adult spine principles and practice. New York: Lippincott-raven; 1991. p. 611-34

3. Lippit AB. The facet joint and its role in spinal pain: management with facet joint injections. Spine. 1984:9(7):746-50.

4. Mooney V, Robertson J. The facet syndrome. Clin Orthop Relat Res. 1976;(115):149-56.

5. Eisenstein SM, Parry CR. The lumbar facet arthrosis syndrome. Clinical presentation and articular surface changes. J Bone Joint Surg Br. 1987;69(1):3-7.

6. Fairbank JC, Park WM, McCall IW, O'Brien JP. Apophyseal injection of local anesthetic as a diagnostic aid in primary low-back pain syndromes. Spine (Phila Pa 1976). 1981;6(6):598-605.

7. Mooney V. Facet joint syndrome. In: Jayson MI. The lumbar spine and back pain. London: Churchill Livingstone; 1992. p.291-306.

\section{RESULTADOS}

Vinte e três pacientes foram inscritos e seguidos no estudo.

Durante o seguimento dos pacientes foram prescritos 35 fármacos com fins analgésicos. Os mais prescritos foram o paracetamol (20 pacientes), o diclofenaco (11 pacientes) e o tramadol (quatro pacientes).

Com relação ao número de analgésicos empregados pelos pacientes, houve uma redução estatisticamente significativa do número de analgésicos empregados ( $p<0,0001)$.

Ao comparar a intensidade da dor medida através da escala visual analógica foi encontrada uma redução significativa ( $p<0,0001)$ da intensidade no pré-operatório e nos controles pósoperatórios em 3, 6 e 12 meses.

\section{DISCUSSÃO}

O método de rizotomia percutânea facetária lombar vem sendo amplamente empregado nos tratamentos de dor crônica lombar. Essa doença de larga incidência na população é caracterizada por dor predominantemente lombar que agrava com a extensão e rotação do tronco ou dor irradiada para membros inferiores com padrão não radicular, geralmente localizada na região glútea e raramente irradiada posteriormente abaixo do joelho.A dor não tem influência com a carga e apresenta alívio durante o repouso ${ }^{2-4}$.

As articulações zigoapofisárias têm interesse clínico por que estão próximas dos foramens intervertebrais, através dos quais os nervos espinhais emergem do canal vertebral. Quando essas articulações apresentam afecções ou são acometidas por processos degenerativos, os nervos espinhais próximos são afetados com frequência. Isto causa dor ao longo dos padrões de distribuição dos dermátomos e espasmos nos músculos derivados dos miótomos correspondentes ${ }^{2-4}$. A denervação das articulações zigoapofisárias lombares é um procedimento usado para tratar a dor lombar possivelmente causada por doença dessas articulações. O processo destrutivo é direcionado aos ramos mediais dos ramos primários dorsais dos nervos espinhais que suprem essas articulações.

Esse método vem sendo utilizado nos casos de dor lombar facetária crônica, caracterizada por dores constantes por, pelo menos, três meses e refratárias ao tratamento conservador corretamente instituído neste período. Trata-se de um método com alta efetividade, baixíssimo índice de complicações e com a necessidade de apenas poucas horas de permanência hospitalar ${ }^{11-13}$.

\section{CONCLUSÃO}

Na presente série prospectiva de casos, a rizotomia facetária por radiofrequência aparece como uma técnica eficaz no tratamento da lombalgia mecânica, encontrando-se uma redução significativa no número de analgésicos empregados e na intensidade da dor.

A evidência acumulada a respeito desta técnica no manejo da dor lombar mecânica sugere um benefício com o seu emprego, sendo necessários novos estudos com boa metodologia e maior volume de pacientes para poder certificar das vantagens desta técnica minimanente invasiva.

8. Shealy CN. Percutaneous radiofrequency denervation of spinal facets. Treatment for chronic back pain and sciatica. J Neurosurg. 1975:43(4):448-51

9. Shealy CN. Facet denervation in the management of back and sciatic pain. Clin Orthop Relat Res. 1976;(115):157-64.

10. Sluijter ME, Koetsveld-Baart CC. Interruption of pain pathways in the treatment of the cervical syndrome. Anaesthesia. 1980;35(3):302-7.

11. Boswell MV Trescot AM, Datta S, Schultz DM, Hansen HC, Abdi S et al. Interventional techniques: evidence-based practice guidelines in the management of chronic spinal pain. Pain Physician. 2007;10(1):7-111.

12. Boswell MV, Colson JD, Sehgal N, Dunbar EE, Epter R. A systematic review of therapeutic facet joint interventions in chronic spinal pain. Pain Physician. 2007;10(1):229-53

13. Niemisto L, Kalso E, Malmivaara A, Seitsalo S, Hurri H. Radiofrequency denervation for neck and back pain. A systematic review of randomized controlled trials. Cochrane Database Syst Rev. 2003;(1):CD004058.

14. Bogduk N, Macintosh J, Marsland A. Technical limitations to the efficacy of radiofrequency neurotomy for spinal pain. Neurosurgery. 1987;20(4):529-35. 